\title{
Etat, firmes et territoires : Jeux d'ombres et de lumières autour du projet de cluster de Mohammedia (Maroc)
}

State, firms and territories: strategies around the cluster of Mohammedia (Morocco)

Staat, Firmen und Territorium : Licht-und Schattenspiele um das Clusterprojekt von Mohamemdia (Marokko)

Jean-Luc Piermay

\section{OpenEdition}

Journals

Édition électronique

URL : http://journals.openedition.org/rge/3030

DOI : $10.4000 /$ rge.3030

ISSN : $2108-6478$

Éditeur

Association des géographes de l'Est

Édition imprimée

Date de publication : 31 décembre 2010

ISSN : 0035-3213

\section{Référence électronique}

Jean-Luc Piermay, «Etat, firmes et territoires : Jeux d'ombres et de lumières autour du projet de cluster de Mohammedia (Maroc) », Revue Géographique de l'Est [En ligne], vol. 50 / 3-4 | 2010, mis en ligne le 13 octobre 2011, consulté le 07 septembre 2020. URL : http://journals.openedition.org/rge/ 3030 ; DOI : https://doi.org/10.4000/rge.3030

Ce document a été généré automatiquement le 7 septembre 2020

Tous droits réservés 


\title{
Etat, firmes et territoires : Jeux d'ombres et de lumières autour du projet de cluster de Mohammedia (Maroc)
}

\author{
State, firms and territories: strategies around the cluster of Mohammedia
}

(Morocco)

Staat, Firmen und Territorium : Licht-und Schattenspiele um das Clusterprojekt von Mohamemdia (Marokko)

Jean-Luc Piermay

\section{Introduction}

1 La question du territoire, au sens de territoire infranational, s'invite depuis peu au Maroc dans le langage public. Les propositions marocaines d'autonomie visant, sinon à résoudre la lancinante question du Sahara occidental, du moins à procurer au Maroc un avantage décisif dans ce conflit auprès des instances internationales, apparaissent comme susceptibles d'entraîner une nouvelle définition des relations de pouvoir entre l'Etat et ses composantes. Le fait est très nouveau. Depuis que le Maroc a recouvré son indépendance politique (1956), après le dépeçage colonial et les menaces qui pesaient sur l'institution du sultanat, la tendance lourde a été à l'affirmation de la fonction royale et à une réunification territoriale que le pouvoir marocain juge encore inachevée. Les politiques de décentralisation n'ont jusque-là pas abouti à mettre en place des collectivités dignes de ce nom. Le roi, le Sahara: deux des trois valeurs intangibles sur lesquelles est fondé le régime (la troisième est l'Islam) font directement référence à un "territoire" qui ne peut être que national.

Une semblable évolution a marqué le discours officiel sur l'économie. Si des politiques d'aménagement ont été menées depuis un demi-siècle, surtout pour atténuer l'extrême 
polarisation des activités sur Casablanca, celles-ci n'ont mobilisé les pouvoirs locaux que marginalement, sinon dans un souci d'accaparement des lots... Mieux, depuis l'avènement de Mohammed VI (1999), le projet de modernisation du pays fait du roi l'acteur majeur de la vie sociale et politique. Le Programme Emergence (2006), réflexion prospective sur l'industrie, a ainsi été conçu par le cabinet international Mac Kinsey, mandaté par le roi, sans concertation avec le milieu local et sans envisager celui-ci autrement que sous la forme de simples localisations. Sur la question des territoires locaux, deux changements significatifs ont toutefois eu lieu à cette occasion: le programme lui-même prévoyait la création de clusters $^{1}$; de plus, a posteriori, les Régions ont été priées de le décliner à leur niveau. Si le deuxième point a surtout montré -sauf à Agadir- le rôle essentiel des autorités déconcentrées, il a paru intéressant d'observer le processus de mise en œuvre des "clusters", à travers des enquêtes et entretiens menés sur celui de Mohammedia, ville importante proche de Casablanca, à cause de sa localisation métropolitaine.

Figure 1 : Localisation des espaces d'accueil industriels autour de Mohammedia et de Casablanca

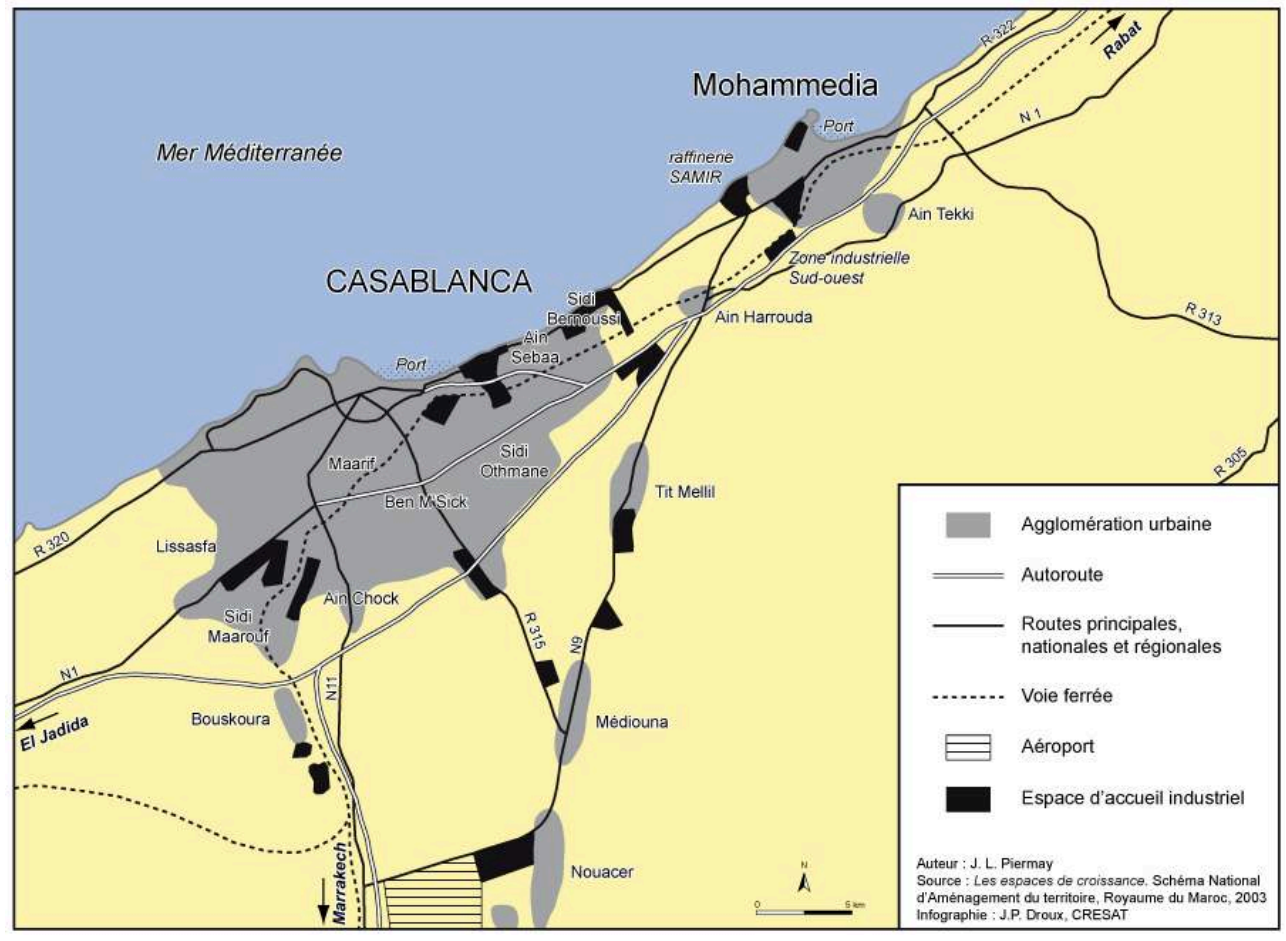

A travers cet exemple précis, la question posée dans cet article est celle de la nature du "moment territorial" que le Maroc connaît actuellement. Il s'agira de comprendre la nature de territoires dont la construction semble si urgente, de la part d'un régime qui s'en est longtemps méfié. Est-il un "espace approprié" (Brunet et al., 1992) ? Est-il un "construit social, économique et politique" résultant d'un processus de coordination des acteurs (Leloup et al., 2005) ? Est-il un "fait social total qui lie les sociétés aux lieux et les sociétés par les lieux" (Piermay, Piveteau, 2006), à l'intersection de l'identité, du politique et des rites (Bonnemaison, 2004) ? En somme, dans la polysémie que recouvre le terme de territoire, polysémie qui peut être source de jeux et de manipulations de la part de certains acteurs, quel est l'usage qui est fait actuellement au Maroc, dans l'irruption soudaine que le mot connaît actuellement? 
4 L'hypothèse est ici que la dimension locale, composante indispensable du concept de territoire, n'est pas une donnée a priori. Comme le territoire (Raffestin, 1986), elle est une construction, dont la définition et même la délimitation constituent un enjeu en soi. Il s'agit donc d'être attentif à un processus de conformation d'une construction politique à un modèle scientifique, dont l'avantage est de donner à cette construction politique un fondement d'irréfutabilité.

\section{Un projet local promu depuis le haut}

5 C'est à l'occasion du Programme Emergence que le projet de cluster "mécatronique" de Mohammedia a d'abord été connu. L'objectif de ce programme était pourtant très différent. Il s'agissait de définir un cadre stratégique pour la politique industrielle du Maroc. Le choix s'est porté sur le développement d'activités exportatrices de haute valeur ajoutée et l'identification d'un certain nombre de secteurs industriels porteurs (aéronautique, automobile, électronique, offshoring), constituant potentiellement des "moteurs mondiaux" de la croissance pour le pays. Ont été ajoutés des secteurs à soutenir, car gros pourvoyeurs d'emplois: agro-alimentaire, transformation des produits de la mer, industrie textile, artisanat. Des localisations propices au développement de chacun de ces secteurs étaient précisées.

Outil de positionnement international de l'industrie marocaine, le Programme Emergence n'avait pas comme finalité d'envisager les articulations de celle-ci avec le milieu local. Toutefois, le terme de "cluster" est utilisé à trois reprises dans le document : à Meknès pour un projet agro-alimentaire, à Agadir pour la transformation des produits de la pêche, et à Mohammedia. Dans l'ensemble du programme, la place de ces projets est très faible. L'électronique, branche concernée par le cluster de Mohammedia, occupe ainsi la plus petite place: 9000 emplois supplémentaires envisagés sur 440000 entre 2009 et 2015, avec 5 lieux d'implantation, dont une "Electronic City" à Tanger. Ce statut spécifique de ces trois opérations au sein du document laisse penser à une genèse plus compliquée que la simple identification d'un créneau porteur par un bureau d'études international. Probablement, l'idée a été reprise par le Programme, lui donnant ainsi une audience accrue. Autre différence notable, contrairement à la grande majorité des projets générés par le Programme Emergence et pour lesquels l'identification des sites d'accueil était en cours en 2009, le cluster de Mohammedia a très vite trouvé son point d'ancrage, la zone industrielle sudouest de la ville, un espace d'accueil de 58 hectares, ouvert en 1989, déjà partiellement occupé.

7 Le projet a eu très vite les honneurs de la presse, comme quelque chose d'original. Les articles étaient assortis de remarques furtives sur ce qui se passait "autrefois" dans la zone industrielle. "Autrefois", c'était avant le passage du roi, en novembre 2000, alors que l'ERAC (Etablissement Régional d'Aménagement et de Construction, devenu holding public Al Omrane) endetté avait vendu les lots aux plus solvables, souvent des non industriels. Avec la visite du souverain et le déblocage d'une somme du Fonds Hassan II pour le développement économique et social, la zone industrielle a commencé une nouvelle vie. Les prix de vente ont été diminués de moitié, et les acquéreurs en règle remboursés en conséquence. Fait exceptionnel au Maroc, les attributaires ont été sommés de mettre en valeur, et les bénéficiaires défaillants de rétrocéder leurs lots, qui 
furent ensuite attribués à de vrais investisseurs. De ce fait, la valorisation de la zone industrielle atteint des taux exceptionnels.

8 Les journaux ont donc chanté les louanges d'une zone industrielle de qualité, qui se remplit avec rapidité et efficacité, offre une forte capacité de création d'emplois et est gérée avec sérieux par une association d'investisseurs à laquelle l'adhésion -fait rare au Maroc- est obligatoire. La poursuite de la trajectoire industrielle semble assurée pour cette vieille ville portuaire et industrielle de Fedala / Mohammedia, née d'une initiative privée en même temps que Casablanca, puis revivifiée par d'importants investissements d'Etat lourds peu après 1960 (raffinerie de pétrole, pétrochimie, centrale thermique). On chante aussi la success story d'un groupe d'entreprises électroniques, qui montent un projet novateur de cluster, en partenariat entre des acteurs locaux et nationaux majeurs, en un site pertinent, proche de Casablanca et de l'autoroute.

9 Instruit par les médias, le chercheur n'en a été que plus étonné par les réalités de terrain. Les statistiques montrent une branche d'activité très minoritaire. En 2004, les $\mathrm{IMM}^{2}$ et IEE regroupées sont en tête du tissu industriel de la préfecture de Mohammedia pour le nombre des établissements $(37 \%)$, en troisième position pour celui des emplois $(29 \%)$ et en dernière position pour la valeur de la production $(4 \%)$ (Azroul, 2006). Les IMM et IEE sont donc éparpillées en de très petits établissements, l'électronique ne faisant pas exception. La ville reste celle des industries lourdes; parmi elles, les toutes puissantes ICP font $71 \%$ de la valeur totale de la production et les IAA $22 \%$.

10 L'enquête annuelle du ministère de l'industrie donne pour 2008 des résultats un peu plus précis pour la zone industrielle sud-ouest, seul espace de la préfecture pour lequel les données sont exhaustives. Sur 94 établissements, 28 sont des IMM $(29,8 \%)$ et 8 seulement (8,5\%) des IEE. En comparaison, les ICP sont $26,6 \%$, les ITC $14,9 \%$, les IAA $11,7 \%$. De leur côté, les enquêtes que j'ai menées auprès de 26 entreprises de la zone industrielle, constituant un échantillon spatial quasi représentatif (novembre 2008), donnent $8 \%$ des établissements et $4,3 \%$ des emplois pour les IEE, $15 \%$ et $16 \%$ pour les IMM, $27 \%$ et $24 \%$ pour les ICP, $23 \%$ et $48,5 \%$ pour les ITC. Il est donc curieux de voir promue au rang de noyau potentiel d'un cluster la branche d'activité la moins représentée localement. Certes, les IMM sont plus nombreuses, mais sous des formes surtout banales (charpentes métalliques, chaudronnerie, menuiserie métallique...), a priori incapables de trouver leur place dans un cluster de mécatronique.

11 L'étonnement du chercheur fut ensuite de constater que la connaissance du dossier par les interlocuteurs diminuait quand il se rapprochait du lieu d'implantation, sauf pour quelques-uns directement engagés dans le projet. Si les services du ministère, à Rabat, en avaient une bonne connaissance, les administratifs de Mohammedia en avouaient leur faible maitrise, et les industriels de la zone ne comprenaient pas la question. Ce halo de mystère, qui faisait douter de la dimension locale du projet, invitait à mieux comprendre la réalité et la nature de celui-ci. 


\section{Un projet malgré tout local}

12 Le caractère "local" du projet de cluster tient surtout à la présence dans la zone industrielle de ses deux piliers, deux chefs d'entreprises. Mais quelle est la consistance de ce "local" très sélectif, aisément camouflé derrière l'échelle nationale?

\section{A. Le projet des promoteurs}

L'idée des promoteurs du projet, conscients d'ouvrir une voie nouvelle pour le Maroc, est de passer d'une situation banale de sous-traitance à la fabrication de produits intégrés complexes. L'objectif n'est donc plus d'accéder à des économies de main d'œuvre, mais d'investir dans la technologie et la valeur ajoutée. Leur ambition est de devenir le sous-traitant privilégié d'un donneur d'ordres international, de préférence installé à proximité. Les entrepreneurs insistent sur les difficultés et la longueur du processus. Ils décrivent leur long cheminement industriel, la complexification progressive de la production, le développement des compétences en ingénierie au sein de leurs entreprises.

Mais ce discours n'est pas seulement celui des promoteurs du projet de cluster. Deux sociétés, non engagées dans celui-ci, ont eu la même analyse. La première revendique quinze ans de passé industriel pour expliquer le saut qualitatif qu'elle est en train d'effectuer; l'autre parle de raccourcis permis par l'intégration dans un groupe international. Toutes deux se déclarent prêtes à franchir le seuil technologique, leur principal défi se situant dans un management capable de montrer la voie. Une troisième entreprise d'électronique de la zone industrielle, qui ne participe pas au projet de cluster, revendique une autre position, le refus de toute R\&D interne au profit du travail à façon pour de grandes entreprises. Il y a donc des stratégies diverses dans les entreprises de la branche et l'absence d'un positionnement économique commun, mais un même souci d'augmenter la valeur ajoutée.

\section{B. Une mobilisation impressionnante d'acteurs}

15 Pour concrétiser cette idée novatrice pour le Maroc, les initiateurs du cluster ont réussi une mobilisation étonnante d'acteurs.

16 A l'origine du projet est une société (A2S Industries), créée en 1997 sous un autre nom, spécialisée dans la fabrication de faisceaux et de cartes électroniques, de même que dans l'intégration de terminaux bancaires. Installée au départ dans le quartier industriel Farah II de Mohammedia, elle est désormais localisée dans la zone sud-ouest, emploie 270 personnes et a connu une forte progression. Elle travaille entièrement pour l'exportation.

17 Autour de cette société, s'est constitué un noyau dur agrégeant quatre autres entreprises ayant des métiers complémentaires, de haute technicité : conception et fabrication de produits électroniques, décolletage et injection plastique, fonderie aluminium sous pression. Toutes sont des PME d'origine française, de Lyon, de la vallée de l'Arve et de Besançon. Toutes continuent à posséder au moins un établissement en France, et l'une d'entre elles est aussi implantée dans d'autres pays. En 2006, ces cinq sociétés ont constitué le GIE GE3M (Groupement électronique, mécatronique et mécanique de Mohammedia), qui a vocation à proposer une offre complète dans la 
spécialité identifiée. Sur cette base, le cluster a été juridiquement créé en 2007, et un directeur nommé.

Mais bien d'autres acteurs ont été partie prenante de ce projet. Des ministres, dont un ancien Premier ministre, ont apporté leur soutien, ainsi que les fédérations nationales de branches concernées. Des conventions ont été signées avec l'Université de Mohammedia et une école de Rabat, de même qu'avec le CETIM (Centre Technique des Industries Mécaniques) de Casablanca; donc, une articulation forte avec le monde de la recherche, tant fondamentale qu'appliquée. De plus, une cinquantaine d'entreprises étrangères, surtout françaises, se seraient déclarées intéressées par le projet; elles font partie du deuxième cercle. En revanche, rares sont les acteurs locaux; parmi eux, le nom d'un industriel s'impose, celui de A. Zouhir, président du groupe SOFMAG et de l'Association de la Zone Industrielle sud-ouest de Mohammedia, ancien président de la Chambre de Commerce, d'Industries et de Services de Mohammedia (CCIS), ancien président de la fédération marocaine des CCIS, ainsi que de celle des pays arabes.

Le projet de cluster butait toutefois sur l'absence d'un acteur jugé indispensable, car seul à pouvoir assurer la pérennité du projet, un donneur d'ordres acceptant de s'installer à côté de ses sous-traitants. Le démarchage a été fait auprès de grandes sociétés françaises et espagnoles, communauté de langues oblige. Trois grandes sociétés, en général déjà liées par des liens de production avec les sociétés du noyau dur, ont été pressenties. L'une d'elles, qui hésitait entre Roumanie, Tunisie et Maroc, serait acquise.

\section{La matérialisation du projet}

Pourquoi le cluster a t-il été installé à Mohammedia? Dans le discours des chefs d'entreprises, pointe le regret que la ville ne réussisse plus la curieuse articulation qu'elle assurait jusque dans les années 1970 entre l'industrie et le tourisme. "Cette ville aurait pu profiter de la congestion du port de Casablanca, elle aurait pu développer des liens avec son arrière-pays. Elle aurait pu donner autre chose que ce que Casablanca peut donner", dit l'un d'eux. "Mais il aurait fallu une vision qu'elle n'a jamais eue". Bien au contraire, la ville s'est trouvée entraînée dans une industrialisation polluante et dans une absorption lente par la grande ville, située à 28 kilomètres. "Elle a subi son développement". Pourtant, de nombreux chefs d'entreprises reconnaissent à la ville des avantages importants, qualité de vie supérieure à Casablanca, tissu industriel dense, zone industrielle bien aménagée, situation favorable, liaison par autoroute. De plus, les entreprises ont bénéficié à Mohammedia d'un foncier plus disponible et moins coûteux. Les liens avec Mohammedia sont aussi ceux qui ont été tissés sur le temps long à l'intérieur du milieu entrepreneurial. Au centre de ce tissu se trouvent les grandes entreprises locales, raffinerie de la SAMIR ${ }^{3}$ et usine pétrochimique de la SNEP ${ }^{4}$. Mais la vision ne serait-elle pas avant tout celle d'A.. Zouhir, qui a passé sa vie professionnelle à Mohammedia?

21 La matérialisation du projet pose la question du foncier. Les terrains pressentis appartiennent à la commune urbaine de Mohammedia. Mais celle-ci n'est dans le montage qu'un acteur secondaire. En fait, après l'affectation à une entreprise qui ne vit pas le jour, les lots en question étaient les seuls disponibles de la zone industrielle. Les acteurs majeurs n'ont pas manqué de moyens de pression pour entraîner l'adhésion de la commune qui n'était acquise ni du côté du président, qui espérait une implantation 
plus lucrative, ni du côté de son opposition, qui redoutait un succès du président... Il ne faudrait pas non plus surestimer l'attractivité de la ville de Mohammedia ; la métropole casablancaise est un argument plus fort pour attirer les industriels, notamment étrangers.

Le projet augmentant en ambition, le terrain de la Commune est vite apparu trop petit. L'idée est maintenant d'y implanter le futur donneur d'ordres et le noyau dur du cluster. Aux autres sous-traitants et aux bâtiments communs, la moitié de la future deuxième tranche de la zone industrielle pourrait être attribuée. Avec le propriétaire, Al Omrane, qui avait aménagé la première tranche, les négociations sont difficiles. Soucieux de rentabilité, le holding réclame des prix jugés "exorbitants" (1 $100 \mathrm{dh} / \mathrm{m} 2)$, proches de ceux pratiqués par le privé pour des terrains industriels. La question de l'aménageur pose aussi problème. Du moins Al Omrane, désormais peu crédible pour les aménagements industriels, n'est pas envisagé.

\section{Les réseaux de relations au cœur du projet}

Entre des acteurs géographiquement dispersés, se pose la question du lien qui rassemble. Ce lien est à trouver dans les relations sociales qu'ont su nouer les différents acteurs.

24 Le patron de la société A2S, M. Tanta, est un des deux piliers du projet. Ce francomarocain né à Casablanca est un industriel bi-national, avec une double résidence, à Mohammedia et à Bordeaux. Après une formation de concepteur de produits, il a derrière lui un passé industriel de quinze ans, d'abord au sein d'un grand groupe. Aujourd'hui, il possède deux sociétés, à Bordeaux et à Mohammedia. Il est donc un passeur, faisant continuellement l'aller et retour, tant physiquement que sur le plan des idées. De plus, trois des quatre autres chefs d'entreprises du noyau dur ont un profil voisin : des Franco-Marocains, habitant ou ayant habité en France, à cheval entre les deux pays.

Les MRE (Marocains Résidant à l'Etranger) ont donc une place importante dans le réseau d'entreprises. L'"étranger" en question se limite à la France et à l'Espagne, et la double communauté de cultures fonde le sentiment d'une vision partagée. Ces MRE se sentent en mesure d'apporter une compétence managériale qui manque au Maroc, la capacité de discuter avec les donneurs d'ordres et d'orienter le travail des ingénieurs et des techniciens. Les rencontres professionnelles, comme le premier salon des Marocains du monde (Nador, 2008), ont joué un rôle dans la constitution d'un réseau, qui ne se limite pas à des Marocains d'origine. Des contacts ont été noués au fil des relations professionnelles et n'ont pas seulement concerné le recrutement d'entreprises. Les liens noués avec le CETIM ont été facilités par la culture binationale de cet organe de contrôle, antenne d'un organisme français, mais installé par des émigrés marocains pour les besoins de l'industrie aéronautique implantée dans l'Aéropôle de Casablanca. Les réseaux sont donc multiples et mêlés.

Le réseau mis en place autour de ces Franco-Marocains ne résout pourtant pas le problème de l'insertion du projet dans le système marocain. Là, une personne a joué le rôle clef, A. Zouhir, dont les multiples casquettes anciennes et actuelles témoignent de l'influence aux échelons local et national. Son passage de treize années à la SAMIR comme Directeur des Etudes et du Développement lui a ouvert de nombreuses portes. Il a réalisé à ce titre l'extension de l'entreprise entre 1970 et 1983 et a retrouvé à cette 
occasion le patron (français) d'une des sociétés chargées des travaux, qu'il avait connu lors de ses études à Toulouse. Pour lui, tout se noue à l'international autour de cette personne. Ensemble, ils créent à Mohammedia une société de montage d'unités industrielles dont il est aujourd'hui le patron, et qui a vite travaillé avec les grandes sociétés locales. A. Zouhir a créé d'autres sociétés; il a acquis une dimension métropolitaine, dans des domaines aussi divers que la margarine, l'électronique, les plastiques, l'engineering. Aujourd'hui, le Groupe SOFMAG qui est le sien a son siège à Casablanca. Mais les relations de A. Zouhir sont aussi d'échelle nationale. C'est lui qui a permis la rencontre des nombreuses personnalités qui ont facilité le montage du cluster. Une remarque importante est que ce réseau n'englobe pas le premier cercle du pouvoir, autour du roi, que l'on retrouve souvent dans les projets industriels majeurs. Il concerne le second, celui qui, autour de l'ancien Premier ministre Driss Jettou, avait trouvé une certaine liberté de mouvement dans le champ du développement économique.

Toujours est-il que l'influence de ce duo est grande. Elle a permis la connexion avec le Programme Emergence et la mise en avant pour Mohammedia des IEE, branche peu représentée jusque-là.

Contrairement à l'idée habituelle du cluster, le projet de Mohammedia constitue une greffe sur un milieu local au rôle limité. Il n'est pourtant pas coupé de celui-ci, puisqu'une proximité physique existe réellement entre des entreprises. Ce n'est pourtant pas celle-ci qui crée la réalité industrielle, mais des réseaux de relations tant industriels que politiques et culturels qui transcendent les échelles et les Etats. De même, l'opacité entretenue au niveau local autour d'un projet pourtant bien monté et consistant, qui se traduit notamment par la rétention des informations envers les acteurs locaux, trahit tout autant un manque de confiance de la part des promoteurs qu'un déficit impressionnant de cohérence territoriale. Cette "manipulation" du local repose la question des objectifs réels du projet de cluster pour ses promoteurs.

\section{Les ressorts d'un projet}

Plusieurs hypothèses permettant de comprendre les logiques profondes du projet de cluster ont été testées autour de questions récurrentes: la faible publicité faite localement à ce projet, la récurrence de la question foncière, les liens entre les principaux protagonistes.

\section{A. Une stratégie industrielle?}

La vision d'une ville de Mohammedia promue au rang de Cité de l'Electronique commence à se diffuser. Mais la vision n'est venue ni de la collectivité locale (elle n'en a pas eu l'idée et n'a pas la force de la promouvoir), ni des acteurs centraux (il a fallu les convaincre). Elle a émergé peu à peu chez quelques chefs d'entreprises.

31 L'idée d'une stratégie d'industrialisation est assez convaincante. Cette volonté de passer d'une situation banale de sous-traitance à une intégration de l'ensemble du processus depuis la conception jusqu'à la réalisation est largement exprimée par les principaux protagonistes; cette idée est fondatrice de leurs discours et se veut susceptible d'être répliquée; pour eux, l'industrie marocaine est appelée à suivre le mouvement qu'ils ont initié. 
32 Mais la stratégie mise en œuvre à Mohammedia est-elle seulement industrielle ? Le doute vient de la présence à proximité immédiate, de sociétés sous-traitantes de même profil, ayant suivi une trajectoire semblable, mais qui n'ont jamais été contactées pour participer au projet et dont les promoteurs du futur cluster sous-estiment d'ailleurs les évolutions technologiques. Probable, la stratégie industrielle n'explique pas tout; elle n'est pas spécifique aux entreprises du cluster et n'est pas non plus spécifiquement ancrée à Mohammedia. Elle n'est pas réellement une "ressource spécifique", dont l'activation fonde le "territoire productif" (Pecqueur, 2005), capable "de générer des rencontres productives" (Perrat, Zimmermann, 2003).

\section{B Une stratégie foncière?}

33 La stratégie de nature foncière est une hypothèse forte, à tester. L'acquisition d'un terrain constitue en effet un blocage essentiel pour les entreprises marocaines, surtout dans la Région du Grand Casablanca, une des plus mal dotées du pays dans la mesure où les pouvoirs publics ont longtemps considéré que la grande ville, déjà très industrialisée, pouvait se débrouiller toute seule. Le projet de cluster n'aurait-il été monté que pour satisfaire un besoin de place?

De fait, la recherche d'un terrain préoccupe depuis deux ans les responsables du projet. Les entreprises du noyau dur, actuellement regroupées dans un seul bâtiment, manquent d'espace. Mieux, les chefs d'entreprises mettent le foncier en bonne place dans leurs discours. Après avoir présenté la philosophie de leur action, ils passent sans trop de transition au blocage majeur qu'il constitue selon eux, avant même d'évoquer les retards industriels du pays. L'un d'eux regrette l'absence de vision sur le foncier au Maroc, de même que le faible nombre de terrains proposés en location. Dans le cas de Mohammedia, il cite pêle-mêle la question du prix des terrains privés, les recours hasardeux (ou tactiques) à la justice et les difficiles négociations avec des paysans qui imaginent que les entreprises industrielles sont riches. Bref, les terrains disponibles pour l'industrie manquent cruellement, face au nombre des demandes (entre 100 et 150 seraient en instance pour la zone industrielle).

Certes, une importante tentative de résolution du blocage foncier est en cours à Casablanca. Le nouveau Schéma Directeur (AUC, 2008) propose une stratégie de long terme, visant à débloquer 5000 hectares (200 ha par an) pour les activités économiques. Ils concernent pour une bonne part Mohammedia, ou plutôt les espaces situés d'une part entre cette ville et Casablanca, d'autre part entre Mohammedia et l'aéroport. De son côté, un plan stratégique du Centre Régional d'Investissement (CRI, 2007), actuellement en préparation ("Business Parc 2020"), doit identifier 7000 ha d'espaces d'accueil pour les entreprises. Mais ces réserves foncières ne sont pas encore acquises et ne peuvent faire oublier les pénuries actuelles, critiques pour des entreprises en forte croissance. Pour autant, la stratégie foncière suffit-elle à expliquer le projet de cluster?

\section{Une stratégie de groupe?}

Entre les trois entreprises du noyau dur déjà présentes sur place, les liens sont étroits, puisqu'elles partagent un même bâtiment, qu'elles ont des clients communs et qu'elles échangent des informations. 

tiers du capital de la société pivot du cluster, A2S, est possédé par le groupe propriétaire d'une des entreprises qu'elle héberge dans ses locaux. Un autre tiers l'est par le groupe SOFMAG, celui d'A. Zouhir. A l'inverse, le patron d'A2S est -ou seraitadministrateur de ces groupes. De même, la liste des entreprises du deuxième cercle comprend plusieurs sociétés appartenant au groupe SOFMAG, certaines étant actuellement installées dans un autre lotissement industriel de Mohammedia. A l'inverse, il est tentant de voir dans la non participation au tour de table de certaines sociétés implantées localement le reflet de relations difficiles avec les responsables du cluster.

L'hypothèse forte est donc que le projet de cluster répond à un objectif de consolidation et de réorganisation du groupe SOFMAG, au moment où le témoin est en train de passer dans la famille Zouhir, du père au fils. La consolidation se fait par le partenariat avec de nouvelles sociétés. Quant à la réorganisation, elle a une évidente composante géographique, autour d'un site de qualité, à Mohammedia, qui concentre déjà deux des trois pôles du groupe, le montage industriel et la mécatronique.

\section{Conclusion}

39 A l'image du "cluster", les termes liés au local et au territoire ont fait une apparition récente dans le champ lexical de l'Etat marocain. Ils se rattachent à un vocabulaire issu du langage scientifique et diffusé par les organisations internationales. Mais la question est moins celle de l'émergence du local -de nombreuses formes de structuration du milieu local existent évidemment au Maroc- que celle du rôle que l'on fait jouer à ce terme.

Une difficulté structurante est au Maroc l'absence d'une réelle décentralisation des pouvoirs. Malgré une Charte communale, plusieurs fois révisée, malgré des lois de décentralisation en faveur des Communes et des Régions, les pouvoirs déconcentrés restent les "autorités locales", et ce depuis les walis en charge des Régions jusqu'au niveau des Communes, en passant par les Gouverneurs en charge des Préfectures et des Provinces. Le projet de cluster de Mohammedia montre une situation différente : ni le Gouverneur de Mohammedia, ni le wali de la Région du Grand Casablanca ne sont des acteurs essentiels. Comme pour les enjeux majeurs du Maroc en termes de développement économique (aménagement de la région de Tanger, modernisation de Rabat, port de Jorf Lasfar...), le pouvoir de décision est aux mains du pouvoir central, avec toutefois la différence importante qu'un Premier ministre a été l'interlocuteur majeur du projet de Mohammedia, tandis que le roi est le pivot incontesté des autres projets.

41 Une deuxième difficulté réside dans l'échelle à laquelle correspond ce "local". Le parcours professionnel de A. Zouhir et son attachement affiché pour Mohammedia ne font pas illusion. Le projet n'est pas à l'échelle de cette ville et l'appellation "Mohammedia" accolée au cluster signifie plus une localisation qu'un enracinement territorial. Les seules organisations capables de réappropriation du projet sont à Casablanca et sont liées au pouvoir déconcentré. Les chefs d'entreprise de Mohammedia eux-mêmes -il en est de même à Rabat, située à une centaine de kilomètres de Casablanca- s'identifient souvent par rapport à la capitale économique du pays, "le lieu où il faut être". De même, l'attractivité de Mohammedia n'est pas la

Revue Géographique de l'Est, vol. 50 / 3-4 | 2010 
sienne propre, mais celle de Casablanca, avec ses infrastructures, son tissu industriel, ses services diversifiés et ses possibilités de relations sociales. Le principal intéressé ne fait pas exception : ses affaires sont à l'échelle de la métropole.

La troisième difficulté réside dans la coalition manifestement réduite d'acteurs proprement locaux. L'absence d'une réelle décentralisation traduit la méfiance du pouvoir central vis-à-vis des élites locales et des instances élues, accusées de gérer leurs clientèles plus que leurs territoires. Dans ces conditions, l'acteur économique devient l'interlocuteur essentiel de l'Etat central, du moins s'il est porteur d'une vision moderniste de la société. Ce faisant, l'Etat central se coupe d'une grande partie des responsables de PME, pour lesquels la firme industrielle constitue un volet -parfois annexe- d'un entreprenariat familial multiforme qui combine courtage, responsabilités politiques, spéculation foncière, voire trafics. Il fait alors plus confiance aux IDE qu'au capitalisme marocain. De même, la faiblesse des couches moyennes vivant de l'industrie et, dans une ville comme Mohammedia, la puissance d'un syndicalisme revendicatif, sont des éléments supplémentaires pour gêner la mise en place d'une coalition locale autour d'un projet commun.

43 Tout indique que le "local" manque de consistance et qu'on lui fait jouer un rôle subalterne. En fait, si la proximité spatiale apparaît quelque peu défaillante, deux formes majeures de proximité sociale sont très apparentes, un réseau économicopolitique et un réseau d'entrepreneurs MRE, dont la rencontre crée le projet. Le premier, ramifiant jusqu'au (ou peut-être "depuis le") pouvoir central, n'est pas sans rappeler les réseaux d'allégeance que le sultan tissait autour de lui jusqu'aux confins de son pouvoir; "toile" que l'on appelait le "Makhzen". Le "local" est ainsi une réalité seconde par rapport au réseau social, une projection du réseau social sur un lieu. Cette logique d'allégeance produit ici des liens incomparablement plus forts qu'une improbable coalition d'acteurs locaux, d'autant plus que le réseau est lié à des ressources internationales. De la part d'un pouvoir central dont l'objectif est la modernisation économique du pays, elle contribue paradoxalement à la reproduction de rapports sociaux et politiques anciens. La nature même du projet montre très clairement un épisode de cette reproduction, un processus de capture d'entrepreneurs MRE, peu enclins jusque-là à investir au pays. En quelque sorte, l'enjeu pour le pouvoir est que cette catégorie sociale devienne une nouvelle élite locale dans le cadre d'une articulation cohérente avec les élites anciennement enracinées dans le système marocain d'allégeance. L'homme clef du projet est bien A. Zouhir, qui articule les deux réseaux sociaux, du pouvoir local et de l'élite extérieure.

Il s'agit maintenant de remonter à la question du territoire. Le "local" en question est sélectif, et c'est précisément pour cela que sa construction est un enjeu. Toutefois, le local n'est ici important que dans la mesure où le territoire, pour être lisible, a besoin d'un ancrage local; on pourrait même dire de manière plaisante que, dans le cas présenté, le local n'est fait que pour être regardé de loin. Pour le reste, l'essentiel est dans la coalition "des acteurs" qu'une des définitions proposées du territoire postulait, mais dans l'existence d'une coalition "d'acteurs" choisis. Ce faisant, le local "partiel" qui a été observé peut ne rester qu'une affaire d'initiés. Que se passerait-il si les indispensables relations de confiance ne s'établissaient pas entre les PME du noyau dur, celles qui viendront à s'installer, le futur donneur d'ordres et les autres partenaires? $\mathrm{Au}$ moins les entreprises du noyau dur auraient-elles acquis des terrains de qualité, et le holding se serait-il consolidé dans d'excellentes conditions! La construction du 
territoire par le haut court le risque de n'être qu'un leurre, mais est-ce réellement un risque quand la valeur ajoutée du "territoire" se mesure en termes d'image?

\section{BIBLIOGRAPHIE}

Agence Urbaine de Casablanca (AUC), 2008, Schéma Directeur d'Aménagement Urbain (SDAU) du Grand Casablanca. Synthèse, Casablanca, octobre 2008, 25 p.

Azroul M., 2006, Diagnostic territorial et identification de projets de SPL : cas du territoire de Mohammedia, mémoire pour l'accès au grade d'ingénieur en chef, ministère de l'industrie, du commerce et de la mise à niveau de l'économie, $64 \mathrm{p}$.

Bonnemaison J., 2004, La géographie culturelle. Cours de l'Université de Paris IV-Sorbonne, Paris, Editions du CTHS, $152 \mathrm{p}$.

Brunet R., Ferras R. et Théry H., 1992, Les mots de la géographie, dictionnaire critique, Montpellier/ Paris, Reclus/La Documentation Française, 470 p.

Centre Régional d'Investissement (CRI) du Grand Casablanca, 2007, « Immobilier d'entreprise. Le Grand Casablanca prépare son Plan Business Parc 2020 », Casa Invest, février 2007, p.9-17.

Leloup F. et al., 2005, « La gouvernance territoriale comme nouveau mode de coordination territoriale? », Géographie, économie, société, n4/2005, Vol.7, p.321-332.

Pecqueur B., 2005, «Le développement territorial : une nouvelle approche des processus de développement pour les économies du Sud », in Antheaume B., Giraut F., Le territoire est mort, Vive les territoires, Paris, IRD Editions, p.295-316.

Perrat J., Zimmermann J-B., 2003, « Stratégies des firmes et dynamiques territoriales ", in Dupuy C., Burmesiter A., Entreprises et territoires, les nouveaux enjeux de la proximité, Paris, La Documentation française, p.15-32.

Piermay J-L., Piveteau A., 2006, « Du Sénégal au Maroc. Pertinence et impertinence des territoires productifs ", Alfa, p.89-101.

Piermay J-L., Piveteau A., 2009, «L'impensé du "local" dans le Programme Emergence du Maroc. Le cas de Tanger ", in Mezouaghi M., Les localisations industrielles au Maghreb ; attractivité, agglomération et territoires, Tunis - Paris, IRMC-Karthala, p.209-232.

Raffestin C., 1986, «Ecogénèse territoriale et territorialité », in Auriac F., Brunet R., Espaces, jeux et enjeux, Paris, Fayard et Fondation Diderot, Nouvelle Encyclopédie des Sciences et des Techniques, p.173-186.

\section{NOTES}

1. Le terme de "cluster" est celui utilisé par les autorités. A la même époque, le ministère de l'aménagement du territoire, de l'eau et de l'environnement lançait une série d'études (qui n'eut pas de suite) sur les Systèmes productifs localisés (SPL), ce qui montre assez, ne serait-ce qu'à 
travers les différences de termes, les rivalités qui peuvent exister entre les instances officielles du pays.

2. IMM : Industries mécaniques et métallurgiques ; IEE : Industries électriques et électroniques ; ICP : Industries chimiques et para-chimiques; IAA : Industries agro-alimentaires ; ITC : Industries textiles et cuirs.

3. SAMIR : Société Anonyme Marocaine de l'Industrie du Raffinage.

4. SNEP : Société Nationale d'Electrolyse et de Pétrochimie.

\section{RÉSUMÉS}

A partir d'investigations de terrain, l'article s'interroge sur la réalité du projet de "cluster" de Mohammedia (Région du Grand Casablanca), prévu dans le Programme Emergence de croissance de l'industrie marocaine. Il pose donc la question du "local" et du "territoire", ou plutôt celle du rôle que l'on fait jouer à ces concepts, dans la politique industrielle du Maroc. Ce projet se révèle être une opération de regroupement d'entrepreneurs, notamment marocains, installés à l'étranger, opération incertaine mais fortement soutenue par le pouvoir central. Non pas local, à l'exception de l'intervention de rares notables, le projet est en fait piloté par le haut, résultat d'une double stratégie, stratégie moderne d'attraction d'IDE et stratégie traditionnelle d'intégration de nouvelles élites dans les réseaux d'allégeance au pouvoir. Certes coalition d'acteurs, mais d'acteurs choisis, le "territoire" n'est alors qu'une tentative de maîtrise des dynamiques locales par le haut.

From field inquiries, this article considers the reality of the project of cluster, which the Moroccan Emergence Programme for industrial growing has planned in Mohammedia, near Casablanca. It asks the research questions of the "local" and the "territory", better the question of the role that one would like these concepts play in the industrial policy. In fact, the project aims to gather entrepreneurs established in foreign countries, namely Moroccans; the operation is unsure, but strongly supported by State. Not local, except some notables' involvement, the project results from a double strategy, a modern one for attracting foreign investments, a traditional one for including new elites in the kingdom's allegiance networks. The cluster is well an actors' coalition, but of chosen and not local actors; it is only an attempt of the central authorities to control the local dynamics.

Nach Geländebeobachtungen untersucht der Artikel die Realität des Clusterprojekts von Mohammedia (Région Gross Casablanca), der im Programm Entwicklung der marokkischen Industrie vorgesehen wird. Er stellt die Frage des Standorts und des Territoriums, oder eher der Rolle, die diesen Konzepten in der Industriepolitik von Marokko gespielt wird. Dieses Projekt erweist sich als einen Zusammenschluss von Unterhehmern, insbesondere aus Marokko, aber im Ausland etabliert. Das Projekt ist ein unsicheres Geschäft, das von der Zentralmacht unterstützt wird. Mit Ausnahmen einiger hochangesehenen Persönlichkeiten wird es nicht lokal unterstützt, sondern von Oben geführt. Es ist das Ergebnis einer Doppelstrategie: eine moderne Strategie, um ADI anzuziehen und eine traditionnelle Strategie zur Integration von neuen Eliten in Netzen, die in der Nähe der Zentralmacht sind. Es handelt sich um ein Bündnis Akteurer, die gewählt wurden und das Territorium ist lediglich ein Versuch, die lokalen Dynamiken vom Oben zu meistern. 
INDEX

Mots-clés : cluster, Etat, Maroc, politique industrielle, territoire

Keywords : cluster, industrial policy, Morocco, State, territory

Schlüsselwörter : Cluster, Industriepolitik, Marokko, Staat, Territorium

\section{AUTEUR}

JEAN-LUC PIERMAY

Professeur à l'Université de Strasbourg - CRESS, EA 1334 - UFR de Géographie - 3, rue de

l'Argonne - 67083 STRASBOURG Cedex - jlp@unistra.fr 